\title{
Fluorescence Reporter in Staphylococcus aureus as a Useful Tool for Studying L-forms and Virulence
}

\author{
Yuanyuan Xu, ${ }^{1}$ Li Wang, Xudong Sun, ${ }^{1}$ Xiaogang Xu, ${ }^{2}$ Ting Hu, ${ }^{3}$ Bo Dong, ${ }^{3}$ Tao Jing, Jian Han, ${ }^{1,}$ and \\ Ying Zhang, ${ }^{1,4, *}$ \\ ${ }^{1}$ Department of Pathogenic Biology, School of Basic Medical Sciences, Lanzhou University, Lanzhou, China \\ ${ }^{2}$ Institute of Antibiotics, Huashan Hospital, Fudan University, Shanghai, China \\ ${ }^{3}$ Grade 2011, School of Clinical Medicine, Lanzhou University, Lanzhou, China \\ ${ }^{4}$ Department of Molecular Microbiology and Immunology, Bloomberg School of Public Health, Johns Hopkins University, Baltimore, MD, USA \\ Corresponding author: Jian Han, Department of Pathogenic Biology, School of Basic Medical Sciences, Lanzhou University. 199 N. Donggang West St, Lanzhou, China. Tel/Fax: \\ +86-13893104956, E-mail: hanj@lzu.edu.cn \\ Corresponding author: Ying Zhang, Department of Molecular Microbiology and Immunology, Bloomberg School of Public Health, Johns Hopkins University, 615 N. Wolfe \\ Street, Baltimore, USA. Tel: +1-4106142975, Fax: +1-4109550105, E-mail: yzhang@jhsph.edu
}

Received 2016 August 14; Accepted 2017 July 18.

\begin{abstract}
Background: Staphylococcus aureus is a prominent pathogen responsible for human pyogenic and nosocomial infections. Although numerous studies on S. aureus Lforms and virulence are available in the literatures, there are still various impediments in the research methods employed.

Objectives: This study aimed to perform fluorescence reporter in studying S. aureus L-form and its virulence, and wild strain virulence.

Methods: Newman-pCM29 strain which can express green fluorescent protein (GFP) was constructed and used to detect S. aureus L-form formation and virulence in vitro and in vivo.

Results: "Fried egg" like L-form colonies of GFP reporter S. aureus and the dense core of the colonies embedded into soft agar can be observed obviously. GFP reporter can contribute to preparing L-form suspension for injection and track the protoplasts and reverted cells in lesions of mice. GFP reporter, also, can help to detect S. aureus wild strain distribution and inflammation scope in lesions, and the fluorescence intensity can be used as an effective surrogate to represent the bacterial quantity in lesions. Fluorescence can improve to distinguish the colonies of varying sizes derived from the injected bacteria or from contaminants.

Conclusions: Fluorescence reporter in S. aureus is a powerful tool which can provide new insight into S. aureus L-form formation in vitro and its fate in vivo, and wild strain virulence in vivo in a non-traditional manner.
\end{abstract}

Keywords: Fluorescence Reporter, GFP, L-Form, Virulence, Staphylococcus aureus

\section{Background}

Staphylococcus aureus is a prominent pathogen responsible for human and animal pyogenic infections including pneumonia, endocarditis (1), mastitis (2), osteomyelitis (3), pyemia (4), skin infections, food-borne diseases (5), as well as nosocomial infections (6). L-forms are bacteria lacking cell wall which were first discovered by Emmy Klieneberger in 1935 (7). Staphylococcus aureus can be induced to unstable cell-wall-deficient L-form in vitro $(8,9)$ or in vivo $(10,11)$ which can still possess parental pathogenicity and cause septicemia, bovine mastitis, as well as chronic and latent lung infections (10-12).

Besides the lack of a cell wall, the unstable L-forms present unique characteristics such as a variety of morphological types, slow growth and proliferation, and formation of small "fried egg" colonies $(9,13)$. Although the core areas of the "fried egg" colonies can be observed under the surface of induced soft agar medium using a light microscope (13), they always appear vague owing to their translucent property. As a result, the detection of $S$. aureus L-forms and their colonies is difficult. Furthermore, the unstable L-forms are difficult to be induced in liquid medium and determination of their concentration by scraping the "fried egg" colonies and suspending them in buffer is challenging. In addition, owing to their atypical shape and regressed properties, there is a dilemma of L-forms tracking when injected in vivo. Thus, these problems hinder the advancement in research on the virulence and immunogenicity of $S$. aureus L-forms.

Staphylococcus aureus possesses a variety of virulence factors such as microcapsules and toxins (14) which contribute to its pathogenicity $(15,16)$. Numerous in vivo assays for the detection of virulence as well as analysis of virulence factors and regulation mechanisms of both wildtype and mutant $S$. aureus are available in the literature (17-19). These assays usually involve mice infected with a specific concentration of $S$. aureus subcutaneously (18), intraperitoneally (19), or intravenously (20). The mice would be killed after a certain time to collect lesions. The samples collected are used for pathological detection after hematoxylin and eosin ( $\mathrm{H}$ and $\mathrm{E}$ ) staining and for $\mathrm{CFU} \mathrm{l}^{-1}$ (CFU: Colony Forming Unit) enumeration after homogenization, serial dilution, plating, and incubation at $37^{\circ} \mathrm{C}$. Although the abscesses and pathogenic bacteria in the lesions could be detected using these methods, the bacterial distribution in the lesions could not be determined. In addition, 
the different size and shape of the colonies on the plate reduce enumeration accuracy.

\section{Objectives}

Pang et al. developed the plasmid pCM29 which can express green fluorescence protein (GFP) to accurately monitor the fate of ingested S. aureus within the polymorphonuclear neutrophil phagosomes (21). In the present study, we transformed the plasmid pCM29 into S. aureus Newman strain and obtained Newman-pCM29 strain, which can express GFP and emit very bright signals. Taking advantage of the newly developed GFP reporter strain, we studied $S$. aureus L-form formation, and virulence properties in vitro or in vivo, as well as in rapid throughput assay that utilizes fluorescence-activated cell sorting for quantification, thus providing new insights into the biology and pathogenesis of this organism.

\section{Methods}

\subsection{Animals}

BALB/c mice aged 6 weeks were obtained from animal centre of Lanzhou University, P.R. China.

\subsection{Ethics Statement}

All experimental procedures involving animals were performed according to the institutional ethical guidelines for animal experiments and approved by the ethics committee for animal use at the Lanzhou University. Animal protocols were approved by the Animal Care Committee of Lanzhou University (NO. SCXKGAN2013-0002).

\subsection{Antibiotics}

Penicillin G and chloramphenicol were obtained from Sangon Biotech (Shanghai, China). The stock solutions of antibiotics were freshly prepared, filter-sterilized, and used at appropriate concentrations as indicated.

\subsection{Bacterial Strains and Growth Conditions}

Staphylococcus aureus Newman strain and its derivatives were cultivated in tryptic soy broth (TSB) (Solarbio, China) and tryptic soy agar(TSA) (Solarbio, China) at $37^{\circ} \mathrm{C}$, and the growth medium was supplemented with chloramphenicol (10 $\left.\mu \mathrm{g} \mathrm{mL}^{-1}\right)$ for the bacteria with pCM29 to maintain the plasmid.
3.5. Electroporation of the Passaged Plasmid pCM29 DNA into S. aureus Newman Strain

Plasmid pCM29, which encodes GFP driven by the P1 promoter of staphylococcal accessory regulator A (sarA), was transformed into $S$. aureus Newman strain by electroporation as described $(21,22)$. In brief, S. aureus RN4220 and Newman strain competent cells were prepared. The plasmid pCM29 was introduced into S. aureus RN4220 cells via electroporation (voltage, $2.5 \mathrm{kV}$; resistance, $100 \mathrm{~V}$; capacity, $25 \mathrm{mF}$ ) using the MicroPulser electroporation apparatus (Bio-Rad, USA). Then the bacteria were plated onto TSA containing $10 \mu \mathrm{g} \mathrm{mL}^{-1}$ chloramphenicol and incubated for 24 hours at $37^{\circ} \mathrm{C}$. The colonies were observed under a fluorescence microscope (Nikon 80i, Japan) and green colonies indicated successful transformation. The plasmid pCM29 was extracted from RN4220-pCM29 and then introduced into $S$. aureus Newman competent cells via electroporation and was identified as described earlier.

\subsection{L-Form of S. aureus Newman Strain and Newman-pCM29 Reporter Strain Induction and Identification}

The preparation of L-form induction medium (LIM) with brain-heart infusion (BHI) (Becton Dickinson, USA), sodium chloride, sucrose, magnesium sulfate, calf serum, and penicillin $\mathrm{G}$, and the induction conditions followed our previous report (23). The typical L-form colonies which appeared as "fried egg," were detected using inverted microscope (Nikon GM3, Japan) and fluorescence microscope, and were confirmed using transmission electronic microscope (TEM) (JEM-1230, Japan) according to the manufacturer's instructions. To determine the relationship between the dense core of the "fried egg" colonies and LIM, the LIM with "fried egg" colonies were cut into slices longitudinally (1-mm thickness) using a sterile scalpel, placed on clean slides, and examined under fluorescence microscope.

3.7. S. aureus L-form Suspension Preparation for Virulence Assay in vivo

To prepare S. aureus L-form suspension, the LIM surface with "fried egg" colonies was repeatedly washed with 1 $\mathrm{mL}$ of phosphate-buffered saline (PBS; $10 \mathrm{mmol} \mathrm{L}^{-1}$ sodium phosphate $(\mathrm{pH}=7.4))$. The washed solution was collected and spotted on a slide and observed under fluorescence microscope.

3.8. Mouse Abscess Model with Newman Strain, NewmanpCM29 Reporter Strain, and L-forms

The virulence of S. aureus Newman strain and NewmanpCM29 reporter strain were analyzed using a mouse abscess model as described previously (18). A total of 24 
BALB/c mice were divided into three groups and their right flank fur was removed with depilatory agent after they were anesthetized. The S. aureus log phase cells were prepared by diluting overnight cultures of Newman strain and Newman-pCM29 reporter strain into TSB (1:100 dilution) and incubated at $37^{\circ} \mathrm{C}$ for about 3 hours to a suitable concentration until the optical density at $600 \mathrm{~nm}$ was 0.8 , respectively. Then, the cultures were centrifuged and the pellets were washed twice with PBS and diluted at a ratio of 1:20 in PBS. To this cell suspension, containing approximately 1 - $3 \times 10^{6} \mathrm{CFU}$ of $S$. aureus, an equal volume of autoclaved Cytodex-1 beads (131 - $220 \mu \mathrm{m}$; Sigma, USA) in PBS was added and mixed well. Then, $0.2 \mathrm{~mL}$ of the Newman strain with beads, Newman-pCM29 mutant with beads, and beads only suspension as a control were injected subcutaneously into the shaved flank of mice in the respective groups. After 48 hours, the mice were euthanized and four subcutaneous abscesses in each group were removed and homogenized in $2 \mathrm{~mL}$ of sterilized PBS. Then, the homogenates were serially diluted 10 -fold in PBS, inoculated onto TSA, and incubated at $37^{\circ} \mathrm{C}$ for 24 hours.

The total number of bacteria in the abscesses was determined by examining the colonies on the plate directly and identified under fluorescence microscope. Furthermore, two subcutaneous abscesses in each group were removed and performed with pathological methods. In addition, two subcutaneous abscesses in each group were removed, frozen, and sliced by Frozen slicer (LEICA CM 1900, Germany), and observed under fluorescence microscope and confocal laser scanning microscope (Olympus FLUOVIEW FV1000, Japan). The fluorescence images were collected at an excitation of $488 \mathrm{~nm}$ and emission of $500-550 \mathrm{~nm}$. The fluorescence intensity of different samples were measured under confocal laser scanning microscope and were compared by statistical analysis (Student's t test).

For the L-form virulence assay, a total of $26 \mathrm{BALB} / \mathrm{c}$ mice were divided into two groups. The L-form suspensions of Newman strain and Newman-pCM29 reporter strain were prepared and injected into the mice as mentioned above. After 72 hours and 120 hours, the lesions were collected, frozen, sliced, and observed under fluorescence microscope.

\section{Results and Discussion}

4.1. Fluorescence Reporter Contributed to the Observation Colony and Growth Characteristics of Unstable L-form in S. aureus

Using previously optimized L-form culture conditions (23), we found that both $S$. aureus Newman strain and Newman-pCM29 reporter strain could be induced into unstable L-forms on LIM. The L-forms, which were confirmed by TEM, displayed typical characteristics as we mentioned previously (9), including completely or partially missing cell wall and different morphological types whereas the normal wild-type $S$. aureus Newman strain had thick cell wall and spherical shape (Figure $1 \mathrm{~A}$ and $\mathrm{B}$ ).

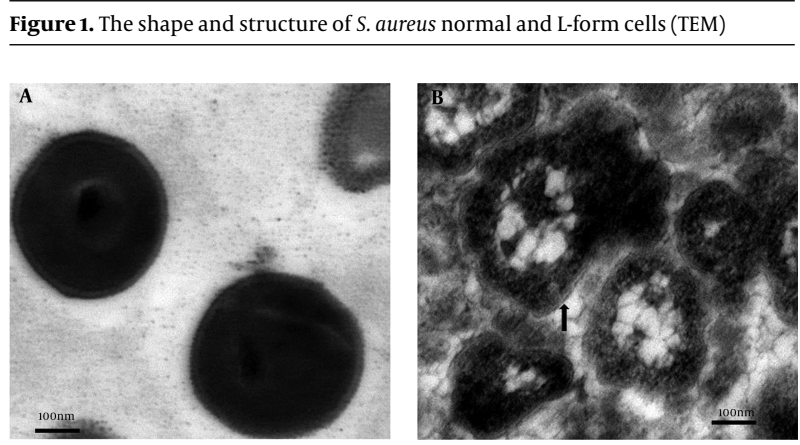

A, Normal S. aureus cells with spherical shape and thick cell wall; B, S. aureus L-forms displaying a variety of morphological types with completely or partially missing cell wall (black arrow).

Both S. aureus Newman strain and Newman-pCM29 reporter strain L-form colonies presented typical "fried egg" shape with round and dense core and thin edge under light microscopy. The thin edge was presented vaguely (Figure 2A). In contrast, under fluorescence microscope, due to the effect of green fluorescence, the "fried egg" colonies of S. aureus Newman-pCM29 reporter strain exhibited vivid shape. The dense core and the thin edges of the colonies were all presented definitely (Figure $2 \mathrm{~B}$ ). This indicates that the promoter of sarA which drives GFP expression in Newman-pCM29 is also expressed in bacterial L-form in addition to its expression in typically cell wall form. In fact, the close similarity of gene expression profiling of bacterial L-form was observed in Escherichia coli (24).

Furthermore, observation of the longitudinally sliced agar culture medium indicated that the dense core of the colonies grew toward the inside of the medium and was embedded into the agar as reported previously $(9,13)$. Owing to the translucent bodies of the $S$. aureus Newman strain L-forms, their embedded dense core of the "fried egg" colonies always have vague shadow under a regular light microscope (Figure 2C). However, with GFP reporter, the size, shape, and position in the agar of the embedded dense core of S. aureus Newman-pCM29 reporter strain Lform, colonies could be observed more clearly under the fluorescence microscope (Figure 2D).

\subsection{Fluorescence Reporter in S. aureus Was Conducive to L-form Pathogenicity Study in vivo}

L-form of S. aureus possesses pathogenicity and can cause diseases under special conditions (10-12). Due to the 
Figure 2. Comparison of the shape and growth properties of the S. aureus Newman strain and Newman-pCM29 reporter strain L-form colonies on LIM
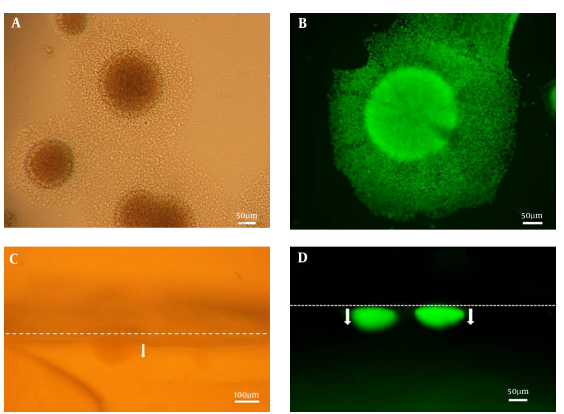

A, The "fried egg" colonies of S. aureus Newman strain L-form; B, The "fried egg" colonies of S. aureus Newman-pCM29 reporter strain L-form under fluorescence microscopy; C, Vague core of S. aureus Newman strain L-form colony embedded into the agar (arrow); D, Definite cores of S. aureus Newman-pCM29 reporter strain L-form colonies embedded into the soft agar (arrows). The dotted lines represent the LIM surface plane.

special characteristics of L-from, there are still many problems and obstacles in its pathogenicity study. Firstly, the suspension for injection to mice is difficult to be prepared. Our previous research showed that $S$. aureus L-form can hardly be induced in liquid medium (data not shown), but it can be induced in soft agar. This was same as reported previously (25). However, the L-form colonies in soft agar exhibit embedded growth and L-form cells to be scraped difficultly from the colonies, resulting in difficulties in preparing bacterial suspensions, as well as determining the final amount of bacteria in the suspension. Secondly, owing to their atypical shape and revertible properties, the L-forms are difficult to be tracked in vivo.

With GFP reporter in S. aureus, it can solve these obstacles. The injected Newman-pCM29 reporter strain L-forms suspension which originated from the LIM surface with "fried egg" colonies by repeatedly washing with PBS were made. With the fluorescence reporter, the Newman-pCM29 reporter strain L-forms could be more clearly quantified in the suspension (Figure 3A) by measuring the fluorescence intensity. Furthermore, when the Newman-pCM29 reporter strain L-forms were injected into mice subcutaneously, the protoplasts and reverted cells in the lesions all emitted green fluorescence (Figure 3B), and the fluorescence reporter contributes to accurate tracking of the pathogenic activities of the S. aureus L-forms in vivo.

\subsection{Fluorescence Reporter in S. aureus Contributes to the Study of S. aureus Virulence in vivo}

In the present study, we used fluorescent intensity measurement and viable bacterial cell counting to represent the bacterial quantification in lesions, which can re-

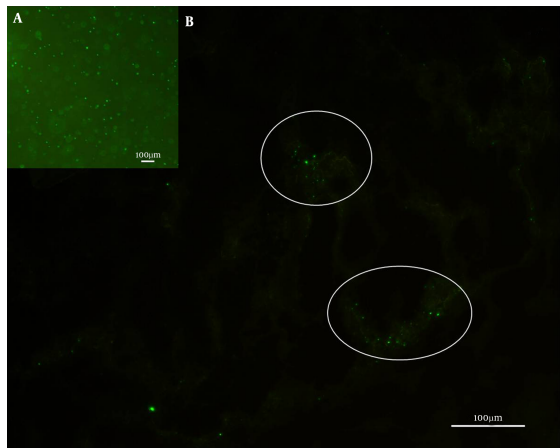

Figure 3. S. aureus Newman-pCM29 L-forms in suspension for mice subcutaneous injection (A) and the fluorescence reported bacteria in mouse subcutaneous lesion after S. aureus Newman-pCM29 L-forms were injected 120 hours (B) (circles)

flect S. aureus virulence in vivo more precisely. Both S. aureus Newman strain and Newman-pCM29 reporter strain caused pyogenic inflammation in mice after subcutaneous injection 48 hours (Figure 4A). Compared with S. aureus Newman strain, Newman-pCM29 reporter strain emitted green fluorescence, and this enabled accurate determination of bacterial distribution and inflammation scope in lesions (Figure 4B and C). The intensity of fluorescence emitted by the $S$. aureus Newman strain infected lesions (19.14 \pm 0.40 ) was significantly lower than that emitted by the S. aureus Newman-pCM29 reporter strain infected lesions $(23.53 \pm 0.48)(P$ value less than 0.05$)$ (Figure $4 D)$. The fluorescence intensity can be used as an effective surrogate to represent the bacterial quantity in lesions when comparing the difference in virulence between the mutants and parent bacteria transformed with fluorescence plasmid in future.

It has been reported that the survival of viable bacterial cells in lesions after the bacteria were injected for a certain period is considered as an important evidence for measuring bacterial virulence $(18,26,27)$. In the present study, colonies of varying sizes were obtained after culturing the homogenized and diluted mice subcutaneous abscesses at $37^{\circ} \mathrm{C}$ (Figure 5A). This result made it difficult to determine whether all the colonies were derived from the injected bacteria or from contaminants. However, with the fluorescence reporter, regardless of their sizes, all the colonies of varying sizes derived from the injected bacteria in the lesions emitted green fluorescence when observed under fluorescence microscope (Figure 5B). This method can help to identify and count the CFUs from the lesions objectively and improve the accuracy.

In the previous research, Yoshioka et al. established a mouse model of soft-tissue infection in the superficial gluteus muscle using bioluminescence imaging (28). The bac- 
Figure 4. S. aureus with GFP reporter in vivo
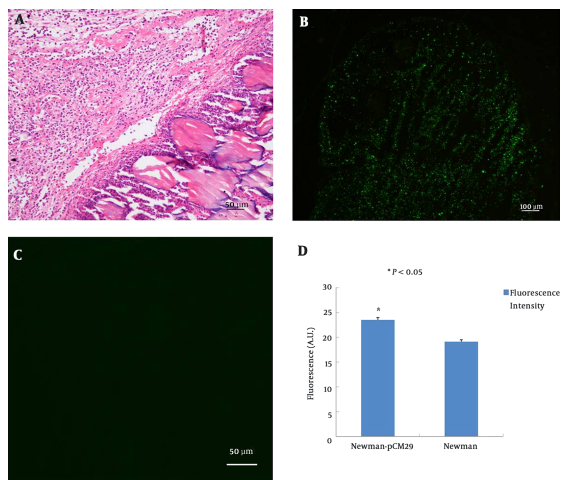

A, The inflammation and abscess formed after subcutaneous injection of $S$. aureus into the mice (H and E stain); B, Distribution of S. aureus Newman-pCM29 reporter strain in lesion observed under fluorescence microscope; C, Lesions infected with S. aureus Newman strain (control) observed under fluorescence microscope; D, The difference in fluorescence intensity between lesions infected with Newman-pCM29 reporter strain and S. aureus Newman strain. A.U., arbitrary unit.

\section{Figure 5. S. aureus viable cells from lesions}
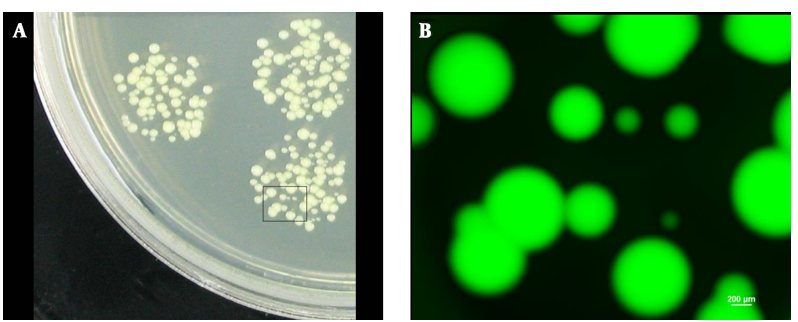

A, Colonies of varying sizes (black box); B, Cells observed under fluorescence microscope. All the colonies inside the black box emitted green fluorescence.

terial quantification in lesions was calculated by analyzing the pixels in Gram-positive areas and bacterial photon intensity. Liese et al. used fluorescence labeled S. aureus to visualize bacterial skin infections in mice (29). In our study, we extended the fluorescence reporter technique to fluorescence intensity measurements and bacterial quantification at the time of virulence determination.

\section{Conclusion}

The fluorescence reporter in S. aureus can contribute to the observation of "fried egg" L-form colonies, performance of L-form in vivo assays, and S.aureus in vivo virulence assays and should serve as a useful tool in research on S. aureus L-form and virulence in future studies.

\section{Acknowledgments}

We wish to express our deep gratitude to Alexander Horswill for providing plasmid pCM29 used in this study.
Our thanks also extend to Zhiwei Wu for confocal laser scanning microscope detection.

\section{Footnotes}

Authors' Contribution: Yuanyuan Xu contributed to data collection, interpretation and analysis, and drafted the manuscript. Li Wang, Xudong Sun, and Tao Jing contributed to data collection and analysis. Xiaogang $\mathrm{Xu}$, Ting $\mathrm{Hu}$, and Bo Dong contributed to experimental operation process. Jian Han and Ying Zhang provided the idea and experimental design of this study and guided the whole study.

Financial Disclosure: The authors declare that no competing financial interest exists.

Funding/Support: This study was supported by national natural Science foundation of China (81571952), the fundamental research funds for the central Universities (lzujbky2016-k02).

\section{References}

1. Li Z, Peres AG, Damian AC, Madrenas J. Immunomodulation and Disease Tolerance to Staphylococcus aureus. Pathogens. 2015;4(4):793815. doi: 10.3390/pathogens4040793. [PubMed: 26580658].

2. Kant R, Taponen S, Koort J, Paulin L, Avall-Jaaskelainen S, Palva A. Genome Sequences of Four Staphylococcus aureus Strains Isolated from Bovine Mastitis. Genome Announc. 2015;3(2) doi: 10.1128/genomeA.00334-15. [PubMed: 25908141].

3. Elasri MO, Thomas JR, Skinner RA, Blevins JS, Beenken KE, Nelson CL, et al. Staphylococcus aureus collagen adhesin contributes to the pathogenesis of osteomyelitis. Bone. 2002;30(1):275-80. doi: 10.1016/S87563282(01)00632-9. [PubMed: 11792597].

4. Powers ME, Bubeck Wardenburg J. Igniting the fire: Staphylococcus aureus virulence factors in the pathogenesis of sepsis. PLoS Pathog. 2014;10(2):e1003871. doi: 10.1371/journal.ppat.1003871. [PubMed: 24550724].

5. Kadariya J, Smith TC, Thapaliya D. Staphylococcus aureus and staphylococcal food-borne disease: an ongoing challenge in public health. Biomed Res Int. 2014;2014:827965. doi: 10.1155/2014/827965. [PubMed: 24804250].

6. Kock R, Friedrich A, On Behalf Of The Original Author Group C. Systematic literature analysis and review of targeted preventive measures to limit healthcare-associated infections by meticillin-resistant Staphylococcus aureus. Euro Surveill. 2014;19(37) [PubMed: 25259535].

7. Klieneberger E. The natural occurrence of pleuropneumonialike organism in apparent symbiosis withStrrptobacillus moniliformis and other bacteria. J Pathol Bacteriol. 1935;40(1):93-105. doi: 10.1002/path.1700400108.

8. Banville RR. Factors affecting growth of Staphylococcus aureus L forms on semidefined medium. J Bacteriol. 1964;87(5):1192-7. [PubMed: 5874541].

9. Han J, He L, Shi W, Xu X, Wang S, Zhang S, et al. Glycerol uptake is important for L-form formation and persistence in Staphylococcus aureus. PLoS One. 2014;9(9):e108325. doi: 10.1371/journal.pone.0108325. [PubMed: 25251561].

10. Tanimoto A, Kitagaki Y, Hiura M, Fujiwara H, Iijima K, Ikawa S. [Methicillin-resistant Staphylococcus aureus forming the fried egg 
appearance colonies isolated from a patient with septicemia]. Rinsho Byori. 1995;43(10):1061-5. [PubMed: 8531391].

11. Owens WE. Isolation of Staphylococcus aureus L forms from experimentally induced bovine mastitis.JClin Microbiol. 1987;25(10):1956-61. [PubMed: 3667916].

12. Michailova L, Kussovsky V, Radoucheva T, Jordanova M, Markova N Persistence of Staphylococcus aureus L-form during experimental lung infection in rats. FEMS Microbiol Lett. 2007;268(1):88-97. doi: 10.1111/j.1574-6968.2006.00567.x. [PubMed: 17168999].

13. Owens WE, Nickerson SC. Morphologic study of Staphylococcus aureus L-form, reverting, and intermediate colonies in situ. J Clin Microbiol. 1989;27(6):1382-6. [PubMed: 2754006].

14. Vandenesch F, Lina G, Henry T. Staphylococcus aureus hemolysins, bicomponent leukocidins, and cytolytic peptides: a redundant arsenal of membrane-damaging virulence factors? Front Cell Infect Microbiol. 2012;2:12. doi: 10.3389/fcimb.2012.00012. [PubMed: 22919604].

15. Cameron DR, Mortin LI, Rubio A, Mylonakis E, Moellering RJ, Eliopoulos GM, et al. Impact of daptomycin resistance on Staphylococcus aureus virulence. Virulence. 2015;6(2):127-31. doi: 10.1080/21505594.2015.1011532. [PubMed: 25830650].

16. Cameron DR, Ward DV, Kostoulias X, Howden BP, Moellering RJ, Eliopoulos GM, et al. Serine/threonine phosphatase Stp1 contributes to reduced susceptibility to vancomycin and virulence in Staphylococcus aureus. I Infect Dis. 2012;205(11):1677-87. doi: 10.1093/infdis/jis252. [PubMed: 22492855].

17. Kim HK, Missiakas D, Schneewind O. Mouse models for infectious diseases caused by Staphylococcus aureus. J Immunol Methods. 2014;410:88-99. doi: 10.1016/j.jim.2014.04.007. [PubMed: 24769066].

18. Ding Y, Onodera Y, Lee JC, Hooper DC. NorB, an efflux pump in Staphylococcus aureus strain MW2, contributes to bacterial fitness in abscesses. J Bacteriol. 2008;190(21):7123-9. doi: 10.1128/JB.00655-08. [PubMed: 18723624].

19. Rauch S, DeDent AC, Kim HK, Bubeck Wardenburg J, Missiakas DM, Schneewind O.Abscess formation and alpha-hemolysin induced toxicity in a mouse model of Staphylococcus aureus peritoneal infection. Infect Immun. 2012;80(10):3721-32. doi: 10.1128/IAI.00442-12. [PubMed: 22802349].

20. Cheng AG, Kim HK, Burts ML, Krausz T, Schneewind O, Missiakas DM.
Genetic requirements for Staphylococcus aureus abscess formation and persistence in host tissues. FASEB J. 2009;23(10):3393-404. doi 10.1096/fj.09-135467. [PubMed: 19525403].

21. Pang YY, Schwartz J, Thoendel M, Ackermann LW, Horswill AR, Nauseef WM. agr-Dependent interactions of Staphylococcus aureus USA300 with human polymorphonuclear neutrophils. J Innate Immun. 2010;2(6):546-59. doi: 10.1159/000319855. [PubMed: 20829608].

22. Bae T, Glass EM, Schneewind O, Missiakas D. Generating a collection of insertion mutations in the Staphylococcus aureus genome using bursa aurealis. Methods Mol Biol. 2008;416:103-16. doi: 10.1007/978-159745-321-9_7. [PubMed: 18392963].

23. Han J, Shi W, Xu X, Wang S, Zhang S, He L, et al. Conditions and mutations affecting Staphylococcus aureus L-form formation. Microbiology. 2015;161(Pt 1):57-66. doi: 10.1099/mic.0.082354-0. [PubMed: 25361600].

24. Glover WA, Yang Y, Zhang Y. Insights into the molecular basis of L-form formation and survival in Escherichia coli. PLoS One. 2009;4(10):e7316. doi: 10.1371/journal.pone.0007316. [PubMed: 19806199].

25. Allan EJ, Hoischen C, Gumpert J. Bacterial L-forms. Adv Appl Microbiol. 2009;68:1-39. doi: 10.1016/S0065-2164(09)01201-5. [PubMed: 19426852].

26. Kwiecinski J, Jin T, Josefsson E. Surface proteins of Staphylococcus aureus play an important role in experimental skin infection. APMIS 2014;122(12):1240-50. doi: 10.1111/apm.12295. [PubMed: 25051890].

27. Tseng CW, Sanchez-Martinez M, Arruda A, Liu GY. Subcutaneous infection of methicillin resistant Staphylococcus aureus (MRSA). J Vis Exp. 2011(48) doi: 10.3791/2528. [PubMed: 21339727].

28. Yoshioka K, Ishii K, Kuramoto T, Nagai S, Funao H, Ishihama $\mathrm{H}$ et al. A novel mouse model of soft-tissue infection using bioluminescence imaging allows noninvasive, real-time monitoring of bacterial growth. PLoS One. 2014;9(9):e106367. doi: 10.1371/journal.pone.0106367. [PubMed: 25184249].

29. Liese J, Rooijakkers SH, van Strijp JA, Novick RP, Dustin ML. Intravital two-photon microscopy of host-pathogen interactions in a mouse model of Staphylococcus aureus skin abscess formation. Cell Microbiol. 2013;15(6):891-909. doi: 10.1111/cmi.12085. [PubMed: 23217115]. 